\title{
REGULARITY AND SENSITIVITY FOR MCKEAN-VLASOV TYPE SPDEs GENERATED BY STABLE-LIKE PROCESSES
}

\begin{abstract}
In this paper we study the sensitivity of nonlinear stochastic differential equations of McKean-Vlasov type generated by stable-like processes. By using the method of stochastic characteristics, we transfer these equations to non-stochastic equations with random coefficients, thus making it possible to use results obtained for nonlinear PDEs of McKean-Vlasov type generated by stable-like processes in previous works. The motivation for studying sensitivity of nonlinear McKean-Vlasov SPDEs arises naturally from the analysis of the mean-field games with common noise.
\end{abstract}

Key words: McKean-Vlasov SPDE, sensitivity, stable-like processes, mean-field games with common noise

2010 Mathematical Subject Classification: 60H15, 35R60, 91A15, 82C22

1. Introduction. In this paper we shall study the well-posedness and sensitivity of the following stochastic partial differential equation of the McKean-Vlasov type generated by stable-like processes,

$$
d\left(f, \mu_{t}\right)=\left(L\left(\mu_{t}\right) f+\frac{1}{2}\left(\sigma_{c o m} \sigma_{c o m}^{T} \nabla, \nabla\right) f, \mu_{t}\right) d t+\left(\sigma_{c o m} \nabla f, \mu_{t}\right) d W_{t}
$$

This equation is written in the weak form meaning that it should hold for all $f \in C^{2}\left(\mathbf{R}^{d}\right)$. Here $x \in \mathbf{R}^{d}, W_{t}$ is $d^{\prime}$-dimensional standard Brownian motion, $\sigma_{\text {com }}$ is a constant $d \times d^{\prime}$ matrix,

$$
L\left(\mu_{t}\right) f(x)=\left(b\left(x, \mu_{t}\right), \nabla\right) f(x)-a(x)|\Delta|^{\alpha / 2} f(x),
$$

is the generator for the stable-like processes in $\mathbf{R}^{d}$ with stability index $\alpha \in$ $(1,2), \mu_{t} \in \mathcal{M}\left(\mathbf{R}^{d}\right)$ (the set of bounded positive Borel measures on $\mathbf{R}^{d}$ ), the drift $b(x, \mu)$ and the scale coefficient $a(x)$ are continuous functions.

(C) Petrozavodsk State University, 2018 
By the usual rule $Y \circ d X=Y d X+\frac{1}{2} d Y d X$, equation (1) rewrites in a more transparent Stratonovich form as

$$
d\left(f, \mu_{t}\right)=\left(L\left(\mu_{t}\right) f, \mu_{t}\right) d t+\left(\sigma_{c o m} \nabla f, \mu_{t}\right) \circ d W_{t} .
$$

Recall that the fractional Laplacian can be expressed via the integral operator

$$
|\Delta|^{\alpha / 2} f(x)=C_{\alpha} \int_{\mathbf{R}^{d}}\left(f(x+y)-f(x)-\frac{(\nabla f(x), y)}{1+|y|^{2}}\right) \frac{d y}{|y|^{d+\alpha}},
$$

with a certain constant $C_{\alpha}$.

The motivation for studying sensitivity of McKean-Vlasov SPDEs (1) and the notation $\sigma_{c o m}$ arise naturally from the analysis of the mean-field games with common noise, in which positions of $N$ agents are governed by the system of SDEs

$$
d X_{t}^{i}=b\left(X_{t}^{i}, \mu_{t}^{N}, u_{t}^{i}\right) d t+\sigma_{c o m} d W_{t}+a^{1 / \alpha}\left(X_{t}^{i}\right) d Y_{t}^{i},
$$

where all $X_{t}^{i}$ belong to $\mathbf{R}^{d}, W_{t}$ is a multidimensional standard Brownian motion referred to as the common noise and $Y_{t}^{i}$ are independent symmetric Lévy processes with the index $\alpha$. The parameters $u_{t}^{i} \in U \subset \mathbf{R}^{m}$ are controls available to the players, trying to maximize their payoffs. The forward component of the forward-backward system of equations expressing of the mean-field game consistency problem is a McKean-Vlasov equation, with the backward component being a Hamilton-Jacobi equation.

There is an extensive literature on properties of McKean-Vlasov SPDEs (see e.g. [1-9, 13, 17, 18] and references therein), based on the diffusion proccesses. The well-posedness of the McKean-Vlasov SPDE was shown in [17] in the class of $L_{2}$-functions, and for measures in [7], though under an additional monotonicity assumption. Our paper is the first one where the McKean-Vlasov type SPDEs for the stable-like underlying Markov processes are analysed.

Sensitivity analysis for nonlinear McKean-Vlasov diffusions and nonlinear stable-like processes, stressing precise estimates of growth of the solutions and their derivatives with respect to the initial data, under rather general assumptions on the coefficients was studied in $[11,13,14]$. The exact estimates become important when treating the extension of these equations having random coefficients, since the noise is usually assumed to be unbounded. 
Our paper is organized as follows. We first summarize the method of stochastic characteristics, see $[13,15,16]$ in the simplified version used here. It is our main tool that allows us to turn a stochastic McKeanVlasov equation into a non-stochastic equation with random coefficients. Then we prove the well-posedness of equation (3). Finally we prove our main results on the smooth sensitivity of this equation with respect to initial data.

The following basic notations will be used:

$C^{n}\left(\mathbf{R}^{d}\right)$ is the Banach space of $n$ times continuously differentiable and bounded functions $f$ on $\mathbf{R}^{d}$ such that each derivative up to and including order $n$ is bounded, equipped with norm $\|f\|_{C^{n}}$ which is the sum of the suprema of the magnitudes of all mixed derivatives up to and including order $n$.

$C_{\infty}\left(\mathbf{R}^{d}\right)$ is the Banach space of bounded continuous functions $f: \mathbf{R}^{d} \rightarrow \mathbf{R}$ with $\lim _{x \rightarrow \infty} f(x)=0$, equipped with sup-norm.

$C_{\infty}^{n}\left(\mathbf{R}^{d}\right)$ is a closed subspace of $C^{n}\left(\mathbf{R}^{d}\right)$ with $f$ and all its derivatives up to and including order $n$ belonging to $C_{\infty}\left(\mathbf{R}^{d}\right)$.

If $\mathcal{M}$ is a closed subset of a Banach space $\mathbf{B}$, then $C([0, T], \mathcal{M})$ is a metric space of continuous functions $t \rightarrow \mu_{t} \in \mathcal{M}$ with distance $\|\eta-\xi\|_{C([0, T], \mathcal{M})}=\sup _{t \in[0, T]}\left\|\eta_{t}-\xi_{t}\right\|_{\mathbf{B}}$. An element from $C([0, T], \mathcal{M})$ is written as $\left\{\mu_{\text {. }}\right\}=\left\{\mu_{t}, t \in[0, T]\right\}$.

$\mathcal{M}\left(\mathbf{R}^{d}\right)$ is the Banach space of finite signed Borel measures on $\mathbf{R}^{d}$.

$\mathcal{M}^{+}\left(\mathbf{R}^{d}\right)$ and $\mathcal{P}\left(\mathbf{R}^{d}\right)$ are the subsets of $\mathcal{M}\left(\mathbf{R}^{d}\right)$ of positive and positive normalised (probability) measures, respectively.

Let $\mathcal{M}_{<\lambda}\left(\mathbf{R}^{d}\right)$ (resp. $\mathcal{M}_{\leqslant \lambda}\left(\mathbf{R}^{d}\right)$ or $\mathcal{M}_{\lambda}\left(\mathbf{R}^{d}\right)$ ) and $\mathcal{M}_{<\lambda}^{+}\left(\mathbf{R}^{d}\right)$ (resp. $\mathcal{M}_{\leqslant \lambda}^{+}\left(\mathbf{R}^{d}\right)$ or $\left.\mathcal{M}_{\lambda}^{+}\left(\mathbf{R}^{d}\right)\right)$ denote the parts of these sets containing measures of the norm less than $\lambda$ (resp. not exceeding $\lambda$ or equal $\lambda$ ).

Let $C^{k \times k}\left(\mathbf{R}^{2 d}\right)$ denote the subspace of $C\left(\mathbf{R}^{2 d}\right)$ consisting of functions $f$ such that the partial derivatives $\partial^{\alpha+\beta} f / \partial x^{\alpha} \partial y^{\beta}$ with multi-index $\alpha, \beta$, $|\alpha| \leqslant k,|\beta| \leqslant k$, are well defined and belong to $C\left(\mathbf{R}^{2 d}\right)$. Supremum of the norms of these derivatives provide the natural norm for this space.

For a function $F$ on $\mathcal{M}^{+}\left(\mathbf{R}^{d}\right)$ or $\mathcal{M}\left(\mathbf{R}^{d}\right)$ the variational derivative is defined as the directional derivative of $F(\mu)$ in the direction $\delta_{x}$ :

$$
\frac{\delta F(\mu)}{\delta \mu(x)}=\left.\frac{d}{d h}\right|_{h=0} F\left(\mu+h \delta_{x}\right)
$$

The same formula defines the variational derivative in the case when $F$ is a Banach space-valued mapping. 
The higher derivatives $\delta^{l} F(\mu) / \delta \mu\left(x_{1}\right) \ldots \delta \mu\left(x_{l}\right)$ are defined inductively.

Let $C^{k}\left(\mathcal{M}_{\leqslant \lambda}\left(\mathbf{R}^{d}\right)\right)$ denote the space of functionals such that the $k$ th order variational derivatives are well defined and represent continuous functions of all variables with measures considered in their weak topology.

Let $C^{k, l}\left(\mathcal{M}_{\leqslant \lambda}\left(\mathbf{R}^{d}\right)\right)$ denote the subspace of $C^{k}\left(\mathcal{M}_{\leqslant \lambda}\left(\mathbf{R}^{d}\right)\right)$ consisting of functionals $F$ such that $\delta^{m} F(\mu) / \delta \mu(.) \ldots \delta \mu(.) \in C^{l}\left(\mathbf{R}^{d}\right)$ for all $m \leqslant k$.

Let $C^{2, k \times k}\left(\mathcal{M}_{\leqslant \lambda}\left(\mathbf{R}^{d}\right)\right)$ be the space of functionals such that their second order variational derivatives are continuous as functions of all variables and belong to $C^{k \times k}\left(\mathbf{R}^{2 d}\right)$ as functions of the spatial variable; the norm of this space is

$$
\|F\|_{C^{2, k \times k}\left(\mathcal{M}_{\leqslant \lambda}\left(\mathbf{R}^{d}\right)\right)}=\sup _{\mu \in \mathcal{M} \leqslant \lambda}\left\|\frac{\delta^{2} F}{\delta \mu\left(\mathbf{R}^{d}\right)}\right\| \|_{C^{k \times k}\left(\mathbf{R}^{2 d}\right)} .
$$

$(f, \mu)=\int f(x) \mu(d x)$ denotes the usual pairing of functions and measures on $\mathbf{R}^{d}$.

2. Stochastic characteristics for commuting groups. Let $L_{t}(\mu)$ be a family of operators defined on a dense subspace $D$ of a Banach space $B$ and depending on $\mu \in B^{*}$ as a parameter. Let $\Omega=\left(\Omega_{1}, \ldots, \Omega_{k}\right)$ be a vector of commuting linear operators $D \rightarrow B$, which generate commuting strongly continuous groups $T_{j}=\exp \left\{t \Omega_{j}\right\}$ in $B$, and hence also weakly continuous groups $T_{j}^{*}=\exp \left\{t \Omega_{j}^{*}\right\}$ in $B^{*}$. Let $W_{t}=\left(W_{t}^{1}, \cdots, W_{t}^{k}\right)$ be a $k$-dimensional standard Brownian motion. We are interested in the weak Stratonovich SDE in $B^{*}$ :

$$
\begin{gathered}
d\left(f, \mu_{t}\right)=\left(L_{t}\left(\mu_{t}\right) f, \mu_{t}\right) d t+\left(\Omega f, \mu_{t}\right) \circ d W_{t} \\
=\left(L_{t}\left(\mu_{t}\right) f, \mu_{t}\right) d t+\sum_{j}\left(\Omega_{j} f, \mu_{t}\right) \circ d W_{t}^{j} .
\end{gathered}
$$

Lemma 1. Under the change of the unknown function $\mu_{t}$ to

$$
\zeta_{t}=\exp \left\{-\Omega^{*} W_{t}\right\} \mu_{t}=\exp \left\{-\sum_{j} \Omega_{j}^{*} W_{t}^{j}\right\} \mu_{t},
$$

equation (6) transfers to a non stochastic PDE with random coefficients:

$$
\frac{d}{d t}\left(f, \zeta_{t}\right)=\left(\tilde{L}_{t}\left(\zeta_{t}\right) f, \zeta_{t}\right)=\left(L_{t}^{\text {dress }}\left(\exp \left\{\Omega^{*} W_{t}\right\} \zeta_{t}\right) f, \zeta_{t}\right),
$$

with

$$
L_{t}^{\text {dress }}(\mu) f=\exp \left\{\Omega W_{t}\right\} L_{t}(\mu) \exp \left\{-\Omega W_{t}\right\} f,
$$




$$
\tilde{L}_{t}\left(\zeta_{t}\right)=L_{t}^{\text {dress }}\left(\exp \left\{\Omega^{*} W_{t}\right\} \zeta_{t}\right) .
$$

Proof. Since the Stratonovich differential is subject to the usual rules of calculus, we have

$$
\begin{gathered}
d\left(f, \zeta_{t}\right)=d\left(\exp \left\{-\Omega W_{t}\right\} f, \mu_{t}\right)= \\
=-\left(\Omega \exp \left\{-\Omega W_{t}\right\} f, \mu_{t}\right) \circ d W_{t}+\left.d\left(g, \mu_{t}\right)\right|_{g=\exp \left\{-\Omega W_{t}\right\} f}= \\
=\left(L_{t}\left(\mu_{t}\right) \exp \left\{-\Omega W_{t}\right\} f, \mu_{t}\right) d t=\left(\exp \left\{\Omega W_{t}\right\} L_{t}\left(\mu_{t}\right) \exp \left\{-\Omega W_{t}\right\} f, \zeta_{t}\right) d t,
\end{gathered}
$$

yielding (7). An alternative proof can be given by first rewriting equation (7) in the Ito form and then perform the transformation using Ito's formula.

As we are mostly interested in the sensitivity, let us formulate the corresponding abstract result, which is a direct consequence of Lemma 1 . Let us concentrate on the case when $B=C_{\infty}\left(\mathbf{R}^{d}\right)$ and $B^{*}=\mathcal{M}\left(\mathbf{R}^{d}\right)$, where the derivatives can be written in terms of the variational derivatives.

Lemma 2. Let equation (7) be well posed and its solutions $\zeta_{t}$ depend smoothly on the initial condition in the sense that the variational derivatives $\delta \zeta_{t} / \delta \zeta_{0}(x)$ and $\delta^{2} \zeta / \delta \zeta_{0}(x) \delta \zeta_{0}(y)$ exist as signed measures and are norm bounded and weak continuous functions of $x$ and $y$. Then the solutions $\mu_{t}=\exp \left\{\Omega^{*} W_{t}\right\} \zeta_{t}$ to equation (3) also depend smoothly on the initial condition $\mu_{0}=\zeta_{0}$ and the variational derivatives are given by the formulas

$$
\begin{gathered}
\left(f, \frac{\delta \mu_{t}}{\delta \mu_{0}(x)}\right)=\frac{\delta}{\delta \mu_{0}(x)}\left(f, \mu_{t}\right)=\frac{\delta}{\delta \zeta_{0}(x)}\left(\exp \left\{\Omega W_{t}\right\} f, \zeta_{t}\right), \\
\left(f, \frac{\delta^{2} \mu_{t}}{\delta \mu_{0}(x) \delta \mu_{0}(y)}\right)=\frac{\delta^{2}}{\delta \zeta_{0}(x) \delta \zeta_{0}(y)}\left(\exp \left\{\Omega W_{t}\right\} f, \zeta_{t}\right) .
\end{gathered}
$$

3. A well posedness result. In this section we shall study the well-posedness of equations (1) or (3).

Equation (3) is an example of equation (6) with

$$
\Omega v(x)=\left(\sigma_{c o m}, \nabla\right) v(x)=\left\{\Omega^{j} v(x)\right\}=\left\{\sum_{k} \sigma_{c o m}^{j k} \nabla_{k} v(x)\right\} .
$$

Operator $\Omega$ has the dual operator

$$
\Omega^{\prime} v(x)=-\Omega v(x)=\left(-\sigma_{c o m}, \nabla\right) v(x),
$$


and generate $d^{\prime}$ semigroups

$$
T_{j}(t) v(x)=v\left(x+\sigma_{c o m}^{j .} t\right)=v\left(x_{1}+\sigma_{c o m}^{j 1} t, \ldots, x_{d}+\sigma_{c o m}^{j d} t\right),
$$

solving the Cauchy problems for the equations

$$
\frac{\partial v}{\partial t}(t, x)=\sum_{k} \sigma_{c o m}^{j k} \frac{\partial v}{\partial x_{k}}(t, x) .
$$

These semigroups commute and define the action $T\left(t_{1}, \ldots, t_{d^{\prime}}\right)$ of $\mathbf{R}^{d^{\prime}}$ on $\left(\mathbf{R}^{d}\right)$ by the formula

$$
T\left(t_{1}, \ldots, t_{d^{\prime}}\right): v(x) \mapsto v\left(x+\sum_{j} \sigma_{c o m}^{j} t_{j}\right)
$$

According to Lemma 1, equation (3) rewrites in terms of the measures $\zeta_{t}=T^{*}\left(-W_{t}\right) \mu_{t}$ as equation (7)

$$
\frac{d}{d t}\left(f, \zeta_{t}\right)=\left(\tilde{L}_{t}\left(\zeta_{t}\right) f, \zeta_{t}\right)=\left(L_{t}^{d r e s s}\left(\zeta_{t}\left(x-\sigma_{\text {com }} W_{t}\right)\right) f, \zeta_{t}\right),
$$

with

$$
\begin{gathered}
L_{t}^{\text {dress }}(\mu) f(x)=\exp \left\{\Omega W_{t}\right\} L(\mu) \exp \left\{-\Omega W_{t}\right\} f(x)= \\
=\left(b\left(x+\sigma_{\text {com }} W_{t}, \mu\right), \nabla\right) f(x)-a\left(x+\sigma_{\text {com }} W_{t}\right)|\Delta|^{\alpha / 2} f(x)
\end{gathered}
$$

and

$$
\begin{gathered}
\tilde{L}_{t}\left(\zeta_{t}\right) f(x)=L_{t}^{\text {dress }}\left(\zeta_{t}\left(x-\sigma_{\text {com }} W_{t}\right)\right) f(x)= \\
=\left(\tilde{b}\left(W_{t}, x, \zeta_{t}\right), \nabla\right) f(x)-\tilde{a}\left(W_{t}, x\right)|\Delta|^{\alpha / 2} f(x),
\end{gathered}
$$

where

$$
\begin{gathered}
\tilde{b}\left(W_{t}, x, \zeta_{t}\right)=b\left(x+\sigma_{c o m} W_{t}, \zeta_{t}\left(x-\sigma_{c o m} W_{t}\right)\right), \\
\tilde{a}\left(W_{t}, x\right)=a\left(x+\sigma_{c o m} W_{t}\right) .
\end{gathered}
$$

We will use several regularity assumptions for the function $b(x, \mu)$ depending on $x$ and $\mu \in \mathcal{M}\left(\mathbf{R}^{d}\right)$. For all practical purposes the dependence of $b$ on $\mu$ is expressed in terms of certain integral functionals, i. e. is of the type

$$
\begin{gathered}
b(x, \mu)=g\left(x, I_{1}, \ldots, I_{\rho}\right), \\
I_{m}=\int_{\mathbf{R}^{d l_{m}}} B_{m}\left(x, y_{1}, \ldots, y_{l_{m}}\right) \mu\left(d y_{1}\right) \cdots \mu\left(d y_{l_{m}}\right),
\end{gathered}
$$


with functions $B_{m}$ which are symmetric in $y$, for which all variational derivatives belong to the same class:

$$
\begin{aligned}
\frac{\delta b(x, \mu)}{\delta \mu(z)}=\sum_{m} \frac{\partial g}{\partial I_{m}}( & \left.x, I_{1}, \ldots, I_{\rho}\right) l_{m} \int_{\mathbf{R}^{d\left(l_{m}-1\right)}} B_{m}\left(x, z, y_{2}, \ldots, y_{l_{m}}\right) \times \\
& \times \mu\left(d y_{2}\right) \cdots \mu\left(d y_{l_{m}}\right),
\end{aligned}
$$

and hence all regularity conditions can be rewritten in terms of the usual smoothness of functions $B_{m}$.

Let us introduce the following conditions:

(C1) function $a(x) \in C_{\infty}^{2}\left(\mathbf{R}^{d}\right)$ and satisfies the inequalities

$$
M^{-1} \leqslant a(x) \leqslant M
$$

for all $x \in \mathbf{R}^{d}$ and a constant $M>1$;

(C2) function $b(x, \mu)$ is continuous and bounded on $\mathbf{R}^{d} \times \mathcal{M}\left(\mathbf{R}^{d}\right)$, $b(., \mu) \in C^{2}\left(\mathbf{R}^{d}\right)$, and $b$ is Lipshitz continuous as a function of $x$, uniformly in other variables;

(C3) the first and second order variational derivatives of $b(x, \mu)$ with respect to $\mu$ are well defined, bounded and

$$
b(x, .) \in\left(C^{2,1 \times 1} \cap C^{1,2}\right)\left(\mathcal{M}_{\leqslant \lambda}\left(\mathbf{R}^{d}\right)\right)
$$

for any $\lambda>0$.

Remark 1. If the function $b$ is represented as (14) then the smoothness of the second order variational derivatives of $b(x, \mu)$ can be expressed in terms of the smoothness of the finite dimensional functions $B_{m}$ and $g$.

The well posedness of this nonlinear non-stochastic equation with random coefficients, (7) or (11), follows from the general results on the wellposedness of nonlinear stable-like equations from [10-12,14] (see e. g. Theorem 7.9 of [11] and Proposition 2.5 of [14]). Due to the equivalence of this equation with equation (3) we obtain the following well-posedness result for (3).

Theorem 1. Under assumptions $(C 1)-(C 3)$ and any given $T>0$ the following holds. 
(i) The Cauchy problem for equation (3) is well posed almost surely, that is, for any initial condition $Y \in \mathcal{M}^{+}\left(\mathbf{R}^{d}\right)$ it has a unique bounded nonnegative solution $\mu_{t}(Y)$ such that $\zeta_{t}=\exp \left\{-\Omega^{*} W_{t}\right\} \mu_{t}$ solves (7) and (11).

(ii) For all $t>0,\left\|\zeta_{t}\right\| \leqslant\|Y\|$ and $\zeta_{t}$ have densities with respect to Lebesgue measure. With some abuse notation, we shall denote these densities again by $\zeta_{t}$. They satisfy the mild equation

$$
\begin{gathered}
\zeta_{t}(x)=\int G_{t}(x, y) Y(d y)- \\
-\int_{0}^{t} d s \int \frac{\partial}{\partial y}\left(G_{(t-s)}(x, y), \tilde{b}\left(W_{s}, y, \zeta_{s}\right) \zeta_{s}(y)\right) d y .
\end{gathered}
$$

Consequently, $\left\|\mu_{t}\right\| \leqslant\|Y\|$ and $\mu_{t}$ also have densities, $v_{t}$ and $\mu_{t}(d y)=v_{t}(y) d y \rightarrow Y$ weakly, as $t \rightarrow 0$. If the initial condition $Y$ has a density, $v_{0}$, then $v_{t} \rightarrow v_{0}$ in $L^{1}\left(\mathbf{R}^{d}\right)$. Here $G_{t}(x, y)$ is the dual Green function to the Green function for the operator $a(x)|\Delta|^{\alpha / 2}$.

(iii) For any two solutions $\mu_{t}^{1}$ and $\mu_{t}^{2}$ of (3) with the initial conditions $Y^{1}, Y^{2}$ the estimate

$$
\left\|\mu_{t}^{1}-\mu_{t}^{2}\right\|_{L_{1}\left(R^{d}\right)} \leqslant\left\|Y^{1}-Y^{2}\right\|_{\mathcal{M}\left(R^{d}\right)} C\left(T,\left\|Y^{1}\right\|\right)
$$

holds, with $C$ depending on the bounds of the derivatives in conditions $(C 1)-(C 3)$.

Let us stress that the coefficients of equation (3) are random, but the estimates of growth (17) are deterministic, because of the uniform bounds for coefficients $\tilde{b}$ and $\tilde{a}$. In fact, the constants $C\left(T,\left\|Y^{1}\right\|\right)$ on the r.h.s. of (17) can be naturally expressed in terms of the Mittag-Leffler functions.

4. Sensitivity: first order. In this section we shall study the sensitivity for the nonlinear stochastic McKean-Vlasov equations (3), that is, the derivatives

$$
\xi_{t}(x ; .)=\xi_{t}(x ; .)\left[\mu_{0}\right]=\frac{\delta \mu_{t}}{\delta \mu_{0}(x)}=\left.\frac{d}{d h}\right|_{h=0} \mu_{t}\left[\mu_{0}+h \delta_{x}\right]
$$

and

$$
\eta_{t}(x, z ; .)=\eta_{t}(x, z ; .)\left[\mu_{0}\right]=\delta^{2} \mu_{t} / \delta \mu_{0}(x) \delta \mu_{0}(z) .
$$

By Lemma 2 these derivatives are expressed in terms of the derivatives of the non-stochastic equation (7) or (11). For nonstochastic equations of 
this kind the sensitivity was obtained in our previous paper (see Theorem 3.2 of [14]), which implies the point-wise (for almost all trajectories $W_{t}$ ) sensitivity of (3). However, what is important for applications is to have estimates of growth of the derivatives $\xi, \eta$ in various functional spaces that are either deterministic or at least bounded in expectation. For the case of constant correlations $\sigma_{\text {com }}$ we shall be able to get bounds that are deterministic. To this end, we have to look at the main stages of the proof of the sensitivity of (7) and (11) and to see exactly how the estimates depend on the coefficients. The key point to note is that, since $\tilde{b}$ and $\tilde{a}$ are obtained by the shifting of $b$ and $a$, the assumptions $(\mathrm{C} 1)-(\mathrm{C} 3)$ on $b$ and $a$ are equivalent to the same assumptions on $\tilde{b}$ and $\tilde{a}$ with the same bounds on the norms in all spaces involved.

Theorem 2. Under Conditions (C1)-(C3) the mapping $Y=\mu_{0} \mapsto \mu_{t}$ from Theorem 1 is continuously differentiable in $Y$, so that the derivative (18) is well defined as a continuous function of two variables such that

$$
\sup _{x} \sup _{\mu_{0} \in \mathcal{M}_{\leqslant \lambda}^{+}\left(\mathbf{R}^{d}\right)}\left\|\xi_{t}(x ; .)\left[\mu_{0}\right]\right\| \leqslant C(T, \lambda,\|Y\|)
$$

for $t \in[0, T]$ uniformly for all values of $W_{t}$. Moreover, the variational derivatives $\xi_{t}(x ;$.$) are twice differentiable in x$ weakly, as the functionals on the spaces of smooth functions, that is,

$$
\partial \xi_{t}(x ; .) / \partial x \in\left(C_{\infty}^{1}\left(\mathbf{R}^{d}\right)\right)^{*}, \quad \partial^{2} \xi_{t}(x ; .) / \partial x^{2} \in\left(C_{\infty}^{2}\left(\mathbf{R}^{d}\right)\right)^{*},
$$

and

$$
\begin{gathered}
\left\|\partial \xi_{t}(x ; .) / \partial x\right\|_{\left(C_{\infty}^{1}\left(\mathbf{R}^{d}\right)\right)^{*}} \leqslant C(T, \lambda,\|Y\|), \\
\left\|\partial^{2} \xi_{t}(x ; .) / \partial x^{2}\right\|_{\left(C_{\infty}^{2}\left(\mathbf{R}^{d}\right)\right)^{*}} \leqslant C(T, \lambda,\|Y\|),
\end{gathered}
$$

again uniformly for all values of the noise $W_{t}$.

Proof. By Lemma 2 it is sufficient to prove the result for the mapping $Y=\mu_{0} \rightarrow \zeta_{t}=\exp \left\{-\Omega^{*} W_{t}\right\} \mu_{t}$ solving (7) or (11). As mentioned above, since this equation is not stochastic we can apply the results of paper [14] to derive that all required derivatives are well defined and moreover, $\tilde{\xi}_{t}=\delta \zeta_{t} / \delta \mu_{0}(x)$ itself and its derivatives $\partial \tilde{\xi}_{t}(x ;.) / \partial x, \partial^{2} \tilde{\xi}_{t}(x ;.) / \partial x^{2}$ solve the weak equation

$$
\frac{d}{d t}\left(f, \tilde{\xi}_{t}(x ; .)\right)=\left(\left(\tilde{b}\left(W_{t}, ., \zeta_{t}\right), \nabla\right) f-\tilde{a}\left(W_{t}, .\right)|\Delta|^{\alpha / 2} f, \tilde{\xi}_{t}(x ; .)\right)+
$$




$$
+\iint\left(\frac{\delta \tilde{b}\left(W_{t}, y, \zeta_{t}\right)}{\delta \zeta_{t}(w)} \tilde{\xi}_{t}(x, d w), \nabla f(y)\right) \zeta_{t}(y) d y,
$$

obtained by the formal differentiation of equation (7), (11). The solution to this equation is obtained by considering the last term as the perturbation to the equation defined by the first term, which in its turn is solved by duality from the dual backward equation (in backward time)

$$
\begin{aligned}
\frac{d}{d t} f_{t}(z)= & -\left(\tilde{b}\left(W_{t}, z, \zeta_{t}\right), \nabla\right) f_{t}(z)+\tilde{a}\left(W_{t}, z\right)|\Delta|^{\alpha / 2} f_{t}(z)- \\
& -\int\left(\frac{\delta \tilde{b}\left(W_{t}, y, \zeta_{t}\right)}{\delta \zeta_{t}(z)}, \nabla f_{t}(y)\right) \zeta_{t}(y) d y .
\end{aligned}
$$

We can see now that, due to the uniformity (with respect to the noise) of the norms in (C1)-(C3), all bounds for the solutions to equation (21) obtained in [14] are also uniform completing the proof.

5. Sensitivity: second order. Similarly to Theorem 2 we can now derive the following result on the second order sensitivity for the McKean-Vlasov equation (3).

We obtain for $\tilde{\eta}_{t}=\delta^{2} \zeta_{t} / \delta Y(x) \delta Y(z)$ the weak equation by differentiating equation (21)

$$
\begin{aligned}
& \frac{d}{d t}\left(f, \tilde{\eta}_{t}(x, z ; .)\right)=\left(\left(\tilde{b}\left(W_{t}, ., \zeta_{t}\right), \nabla\right) f-\tilde{a}\left(W_{t}, .\right)|\Delta|^{\alpha / 2} f, \tilde{\eta}_{t}(x, z ; .)\right)+ \\
& \quad+\left(f, q_{t}\right)+\iint\left(\frac{\delta \tilde{b}\left(W_{t}, y, \zeta_{t}\right)}{\delta \zeta_{t}(w)} \tilde{\eta}_{t}(x, z ; d w), \nabla f(y)\right) \zeta_{t}(y) d y
\end{aligned}
$$

with $\left(f, q_{t}\right)$ being given by

$$
\begin{aligned}
& \iint\left(\frac{\delta \tilde{b}\left(W_{t}, y, \zeta_{t}\right)}{\delta \zeta_{t}(w)}, \nabla f(y)\right)\left[\tilde{\xi}_{t}(x ; d y) \tilde{\xi}_{t}(z ; d w)+\tilde{\xi}_{t}(x ; d w) \tilde{\xi}_{t}(z ; d y)\right]+ \\
& \quad+\iiint\left(\frac{\delta^{2} \tilde{b}\left(W_{t}, y, \zeta_{t}\right)}{\delta \zeta_{t}(w) \delta \zeta_{t}(u)}, \nabla f(y)\right) \tilde{\xi}_{t}(x ; d w) \tilde{\xi}_{t}(z ; d u) \zeta_{t}(y) d y
\end{aligned}
$$

which should be satisfied with the vanishing initial condition.

This is the same equation as (21), but with the additional non-homogeneous term $\left(f, q_{t}\right)$. 
The structure of (24) conveys an important message that for this analysis one needs the exotic spaces $C^{2, k \times k}\left(\mathcal{M}_{\leqslant \lambda}^{+}\left(\mathbf{R}^{d}\right)\right)$ introduced in the introduction.

Theorem 3. Under Conditions (C1)-(C3) the mapping $Y=\mu_{0} \mapsto \mu_{t}$ from Theorem 1 is twice continuously differentiable in $Y$, so that the derivative $\eta_{t}(x, z ;$.$) is well defined as a continuous function of three vari-$ ables such that

$$
\sup _{x} \sup _{z} \sup _{\mu_{0} \in \mathcal{M}_{\leqslant \lambda}^{+}\left(\mathbf{R}^{d}\right)}\left\|\eta_{t}(x, z ; .)\left[\mu_{0}\right]\right\| \leqslant C(T, \lambda,\|Y\|)
$$

for $t \in[0, T]$ uniformly for all values of $W_{t}$.

Moreover, the derivatives of $\eta_{t}(x, z ;$.$) with respect to x$ and $z$ of order at most one are well-defined as elements of $\left(C^{2}\left(\mathbf{R}^{d}\right)\right)^{*}$ and

$$
\left\|\frac{\partial^{\gamma}}{\partial x^{\gamma}} \frac{\partial^{\beta}}{\partial z^{\beta}} \eta_{t}(x, z ; .)\right\|_{\left(C^{2}\left(\mathbf{R}^{d}\right)\right)^{*}} \leqslant C(T, \lambda,\|Y\|)
$$

for $\gamma, \beta=0,1$.

Proof. The proof is analogous to that of Theorem 2 and is based on the application of the results of paper [14].

Acknowledgment. The second author's work is supported by the Ministry of Science and Higher Education of the Russian Federation (Grant 1.6069.2017/8.9).

\section{References}

[1] Bardi M., Priuli F. S. Linear-quadratic n-person and mean-field games with ergodic cost. SIAM J. Control Optim., 2014, vol. 52 (5), pp. $3022-3052$.

[2] Basna R., Hilbert A., Kolokoltsov V. N. An Approximate Nash Equilibrium for Pure Jump Markov Games of Mean-field-type on Continuous State Space. Stochastics, 2017, vol. 89 (6-7), pp. 967-993.

DOI: https://doi.org/10.1080/17442508.2017.1297812.

[3] Bensoussan A., Frehse J., Yam P. On the Interpretation of the Master Equation. Stochastic Process. Appl., 2017, vol. 127 (7), pp. 2093-2137. DOI: https://doi.org/10.1016/j.spa.2016.10.004.

[4] Cardaliaguet P. The Convergence Problem in Mean Field Games with Local Coupling. Appl. Math. Optim., 2017, vol. 76 (1), pp. $177-215$.

DOI: https://doi.org/10.1007/s00245-017-9434-0. 
[5] Carmona R. Delarue F. Probabilistic Theory of Mean Field Games with Applications I, II. Probab. Theory Stoch. Model., 2018, vol. 83-84.

[6] Crisan D. McMurray E. Smoothing properties of McKean-Vlasov SDEs Probab. Theory Related Fields, 2018, vol. 171 (1-2), pp. 97-148. DOI: https://doi.org/10.1007/s00440-017-0774-0

[7] Dawson D., Vaillancourt J. Stochastic McKean-Vlasov equations. NoDEA, 1995, vol. 2, pp. $199-229$.

[8] Hu Y., Yaozhong, Nualart D., Song J. A nonlinear stochastic heat equation: Hölder continuity and smoothness of the density of the solution. Stochastic Process. Appl., 2013, vol. 123 (3), pp. 1083-1103.

[9] Huang M., Malhamé R., Caines P. Large population stochastic dynamic games: closed-loop Mckean-Vlasov systems and the Nash certainty equivalence principle. Commun. Inf. Syst., 2006, vol. 6, pp 221-252.

[10] Kolokoltsov V. N. Nonlinear Markov semigroups and interacting Lévy type processes. J. Stat. Phys., 2007, vol. 126 (3), pp. 585-642.

[11] Kolokoltsov V. N. Nonlinear Markov processes and kinetic equations. Cambridge Tracks in Mathematics 182, Cambridge Univ. Press, 2010.

[12] Kolokoltsov V. N. Symmetric stable laws and stable-like jump-diffusions. Proc. Lond. Math. Soc., 2000, vol. 80(3), pp. 725-768.

[13] Kolokoltsov V. N., Troeva M. Regularity and sensitivity for McKean-Vlasov SPDEs. AIP Conf. Proceedings. Proceedings of the 8th Intern. Conf. on Mathematical Modeling (ICMM-2017), 2017, vol. 1907, pp. 030046. DOI: https://doi.org/10.1063/1.5012668.

[14] Kolokoltsov V. N., Troeva M., Yang W. Mean field games based on stablelike processes. Autom. Remote Control, 2016, vol. 77 (11), pp. 2044-2064.

[15] Kolokoltsov V. N., Tyukov A. E. Small time and semiclassical asymptotics for stochastic heat equation driven by Lévy noise. Stochastics and Stochastics Reports, 2003, vol. 75 (1-2), pp. 1-38.

[16] Kunita H. Stochastic flows and stochastic differential equations. Cambridge Studies in Advanced Mathematics. Cambridge Univ. Press, 1997.

[17] Kurtz Th., Xiong J. Particle representations for a class of nonlinear SPDEs. Stochastic Process. Appl., 1999, vol. 83, pp. 103-126.

[18] Nourian M., Caines P. $\varepsilon$-Nash mean field game theory for nonlinear stochastic dynamical systems with major and minor agents. SIAM J. Control Optim., 2013, vol. 51 (4), pp. 3302-3331. 
Received August 24, 2018.

In revised form, December 09, 2018.

Accepted December 11, 2018.

Published online December 13, 2018.

\section{N. Kolokoltsov}

Department of Statistics, University of Warwick

Coventry CV4 7AL UK;

Faculty of Applied Mathematics and Control Processes,

Saint-Petersburg State University, Saint Petersburg, Russia;

Institute of Informatics Problems,

Federal Research Center "Computer Science and Control",

RAS, Moscow, Russia

Email: v.kolokoltsov@warwick.ac.uk

M. S. Troeva

Research Institute of Mathematics, North-Eastern Federal University 58 Belinskogo str., Yakutsk 677000, Russia

Email: troeva@mail.ru 